\title{
Smectic A - Cholesteric Phase Transition Investigated By Small Angle X-Ray Diffraction and Viscosity Measurements
}

\author{
E. L. Duarte ${ }^{1}$, R. Itri ${ }^{1}$, A. R. Sampaio ${ }^{2}$, M. Simões ${ }^{3}$, and A. J. Palangana ${ }^{2}$ \\ ${ }^{1}$ Instituto de Física, Universidade de São Paulo, \\ Caixa Postal 66318, 05315-970 - São Paulo, SãoPaulo, Brazil \\ ${ }^{2}$ Departamento de Física, Universidade Estadual de Maringá, \\ 87020-900 - Maringá, Paraná, Brazil \\ ${ }^{3}$ Departamento de Física, Universidade Estadual de Londrina, 86051-970, Londrina, Paraná, Brazil
}

Received on 30 November, 2001

\begin{abstract}
We have studied the influence of the mean molecular length on the nature of the cholesteric $\left(N^{*}\right)-$ smectic A $(A)$ liquid crystal phase transition for thermotropic compounds of cholesteryl myristate $\left(C_{14}\right)$, cholesteryl nonanoate $\left(C_{9}\right)$, and binary mixtures of $C_{14}-C_{9}$ and cholesteryl caproate $\left(C_{6}\right)$ $-C_{9}$. Mesoscopic properties of these systems, as the distance between smectic layers in the $A$ phase $\left(d_{A}\right)$, the mean molecular length $(\bar{\ell})$ in the $N^{*}$ domain, and the correlation length $(\varepsilon)$, as well as changes along the $N^{*}-A$ phase transition, were determined from the X-ray diffraction data. The results show a decrease in $A$ phase domain and an expansion of the $N^{*}$ domain as the mean molecular length is diminished. Such effect induces a second order $A-N^{*}$ phase transition for a particular mixture of $63.1 \mathrm{~mol} \%$ of $C_{9}$ and $36.9 \mathrm{~mol} \%$ of $C_{6}$, evidenced by viscosity measurements in good agreement with previous density data.
\end{abstract}

\section{Introduction}

The first (discontinuous) and second (continuous) order nature of the liquid crystal phase transitions have been the subject of many works along the last 30 years. In particular, the Smectic $A(A)-N$ ematic $(N)$ or cholesteric $\left(N^{*}\right)$ phase transition was demostrated to be of either first order or second order, depending on the smectic layer thickness and the reduced temperature, according to McMillan's molecular model $[1,2]$. The reduced temperature is defined as [1]: $r=T_{A N} / T_{N I}$, where $T_{A N}$ and $T_{N I}$ represent, respectively, the $A-N$ and $N$-Isotropic $(I)$ phase transition temperatures, also known as McMillan ratio. In a extension of the McMillan's model proposed by Lee et al. [3], the theoretical results point out that the energy associated to the smectic ordering increases with molecular length and $r$, occurring a second order phase transition for $r \leqslant 0.88$. Several calorimetric measurements were performed on binary mixtures (where the molar ratio was varied between two different homologous liquid crystal molecules of distinct lengths) and showed that the reduced temperature can vary from 0.89 to $0.99[4,5]$. In fact, calorimetric measurements performed by two of us on binary mixtures composed of cholesteryl palmitate $\left(C_{16}\right)$, cholesteryl myristate $\left(C_{14}\right)$, cholesteryl nonanoate $\left(C_{9}\right)$ and cholesteryl caproate
$\left(C_{6}\right)$ [6] and $C_{9}$, cholesteryl heptanoate $\left(C_{7}\right)$ and $C_{6}$ [7], that present a $A-N^{*}$ phase transition, gave evidence of a tricritical point around $r \simeq 0.90-0.92$. Recently, we have investigated the possible existence of a second order phase transition at $r \simeq 0.92$ for a particular mixture of $C_{6}-C_{9}\left(63.1 \mathrm{~mol} \%\right.$ of $\left.C_{9}\right)$ through density measurements [8].

It is well known that the average correlation length $(\varepsilon)$ in a second order phase transition diverges continuously in the vicinities of the transition. With a view of investigating the correlation length behavior in our systems, we extended the previous work [8] making use of the X-ray scattering technique to explore the values of $\varepsilon$ within the $N^{*}$ and $A$ phases domain as well as along the $A-N^{*}$ phase transition. In this context, we have studied the same samples previously investigated [8] composed of pure $C_{9}$ and $C_{14}$, and binary mixtures of $C_{14}-C_{9}$ and $C_{9}-C_{6}$. The mean molecular lengths $\left(l_{M}\right)$ are displayed in Table 1 , calculated from the molecular structure taking into account the molar ratio of the compounds employed in the mixtures. The molecular length of each compound was estimated as a

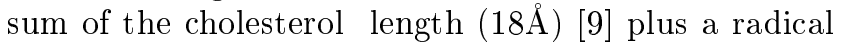
length given by Tanford's formula [10]. 
Table 1: Calculated mean molecular length $\left(l_{M}\right)$, reduced temperature $r=T_{A N^{*}} / T_{I N^{*}}$ reported in [8] and $d_{A}, \bar{\ell}_{A N^{*}}$, determined by X-ray diffraction (see text for details).

\begin{tabular}{|c|c|c|c|c|c|c|}
\hline & \multicolumn{2}{|c|}{ Concentration (mol\%) } & $l_{M}$ & $r$ & $d_{A}$ & $\ell_{A N^{*}}$ \\
\hline & & & $(\AA)$ & & $(\AA)$ & $(\AA)$ \\
\hline$C_{14}$ & 100.0 & 0.0 & 37.2 & $0.984^{(a)}$ & 33.5 & 40.0 \\
\hline$C_{14}-C_{9}$ & 81.5 & 18.5 & 35.9 & 0.975 & 32.0 & 38.1 \\
\hline$C_{14}-C_{9}$ & 63.5 & 36.5 & 35.0 & 0.968 & 31.0 & 37.3 \\
\hline$C_{9}$ & 0.0 & 100.0 & 30.9 & 0.954 & 27.3 & 33.2 \\
\hline$C_{6}-C_{9}$ & 36.9 & 63.1 & 29.6 & 0.920 & 26.2 & 32.1 \\
\hline
\end{tabular}

${ }^{(a)}$ The blue phase was observed between the $N^{*}$ and $A$ phases, in agreement with literature [17]. However, for simplicity due to its narrow domain, the blue phase was not accounted for in the reduced temperature definition.

The variation in concentration implies a change in the mean molecular length and plays an important role as a thermodynamical variable in the study of these systems. The X-ray data furnishes the distance between the smectic layers $\left(d_{A}\right)$ in $A$ phases and the experimental mean molecular length $(\bar{\ell})$ in $N^{*}$ phases from the diffraction peak position, and $\varepsilon$ from the diffraction line width, as shown below. In the literature, few investigations have been reported on the viscosity $(\eta)$ values associated to the $A-N^{*}$ phase transition. In particular, Porter et al.[11] have carried out viscosity measurements as a function of temperature for cholesteryl myristate $\left(C_{14}\right)$ in the $A, N^{*}$ and $I$ phases. The authors showed that the viscosities for both the cholesteric and smectic $A$ phases present a non-Newtonian behavior with a sharp transition between the two mesophases.

\section{Experimental}

\section{II.1 Samples}

Cholesteryl $n$-alkanoate homologous series $C_{14}, C_{9}$ and $C_{6}$ were obtained from Sigma and used without further purification. Calorimetric and X-ray scattering measurements were performed on $C_{14}$ and $C_{9}$ and on three binary mixtures in the following concentrations: $81.5 \mathrm{~mol} \%$ and $63.5 \mathrm{~mol} \% C_{14}$ in the binary mixture of $C_{14}-C_{9}$ and $63.1 \mathrm{~mol} \%$ of $C_{9}$ in the mixture of $C_{9}-C_{6}$.

It should be remarked that only the $C_{14}$ compound presents the crystalline, $A, N^{*}$ and $I$ phases at heating and cooling cycles. The other samples present monotropy in the $A$ phase $[6,7]$. Then, the samples were first heated up to the $I$ phase in a fast way followed by a slower cooling procedure up to the $A$ phase. When the latter was reached (without the appearance of a crystalline phase) the process was reverted, rising again the temperature. Such procedure permitted to acquire the $A-N^{*}$ phase transition data at both cooling and heating cycles.

\section{II.2 Methods}

The X-ray scattering measurements were performed by using a focusing camera in a $18 \mathrm{~kW}$ rotating anode X-ray generator (Rigaku-Denki) with $\mathrm{CuK \alpha}(\mathrm{Ni}$ filter, $\lambda=1.5418 \AA$ ) radiation at $50 \mathrm{kV} \times 200 \mathrm{~mA}$ and an image plate detection system. Samples were acconditioned in sealed $1 \mathrm{~mm}$-thick flat mylar windows, perpendicular to the incident $\mathrm{X}$-ray beam, placed in a copper sample holder in which a temperature control was attached (Euro Control model PC-850A). Further, the sample holder was rotated during the $\mathrm{X}$-ray data acquisition to avoid textures effects (preferential orientation) in the diffraction lines. Data acquisition of three hours was taken for each studied temperature and the temperature step was $2^{\circ} \mathrm{C}$ at both cycles. The spectra registered in the image plate were digitized using a Scanner model STORM 820 with a help of a $P C$ microcomputer.

The 2-D spectra were converted in 1-D (area integration) by using the "X-Ray" program [12], that showed to be an excellent software to analyse this type of image, furnishing the X-ray intensity $\left(I_{o b s}(q)\right)$ as a function of the scattering vector $(q=4 \pi \cdot \sin \theta / \lambda$, where $2 \theta$ is the $\mathrm{X}$-ray scattering angle). The total intensity was normalized by the exposition time and corrected by subtracting a parasitic scattering considered as the low scattering of the $I$ phase. The position and width of the diffraction peak were obtained through fitting a Lorentzian function [13] to the experimental data as:

$$
I_{o b s}(q) \propto\left[\varepsilon^{2} \cdot\left(q-q_{c}\right)^{2}+1\right]^{-1}
$$

where $q_{c}$ is the peak position, and $\varepsilon$ is the correlation length. From $q_{c}$, we obtain the distance between the smectic layers in the $A$ phase: $d_{A}=2 \pi / q_{c}$; and the mean molecular length in the $N^{*}$ phase [14]: $\bar{\ell}=2 \pi(1.229) / q_{c}$. The values of $d_{A}$ and $\bar{\ell}$ presented in the current work, correspond to the mean values obtained at cooling and heating cycles due to their similarity. 
Viscosity measurements as a function of the temperature near the $A-N^{*}$ phase transition were determined by means of a Brookfield Cone/Plate Rheometer model $L V D V-I I I$. The rheometer was calibrated with a standard oil of known viscosity. The sample temperature was controlled by a HetoCB $8-30 E / D T-1$ circulating temperature bath, ensuring that the temperature readings were stable at $0.01 \mathrm{~K}$. A cooling rate of 6 $\mathrm{mKs}^{-1}$ was used during the measurements. Additional measurements were also made as a function of shear rate. The phase transition temperatures were checked by using a polarized light microscope.

\section{Results and discussion}

\section{III.1 X-Ray Scattering}

The X-ray scattering spectra of thermotropic mesophases $A$ and $N^{*}$ present two rings [14]. The first ring appears in a small angle range and is associated to the layer repetition distance in the $A$ phase, and the mean molecular length in the $N^{*}$ phase. The second ring, presented in larger angles, is characteristic of an average distance between the molecules perpendicular to the long axis of the same, with small variation along of the phases.

In this work, we are interested in investigating the variation of the correlation length $(\varepsilon)$ in the two mesophases, as well as along the $A-N^{*}$ phase transition. Therefore, we focus our attention on the first diffraction ring $\left(2 \theta \simeq 2.5^{\circ}\right)$. The features of the $\mathrm{X}$-ray spectra (Fig. 1) of each phase can be summarized as follows:

1. I phase: the X-ray scattering is diffuse (Fig.1-a), characteristic of a disordered distribution of the molecules.

2. $N^{*}$ phase: the X-ray scattering presents a well defined ring (Fig. 1-b), related to the correlation between molecules nearly oriented along a director axis.

3. A phase: the $\mathrm{X}$-ray scattering presents a thinner and stronger ring than that observed in the $N^{*}$ phase (Fig. 1-c), due to a more ordered structural arrangement.

\section{c.}

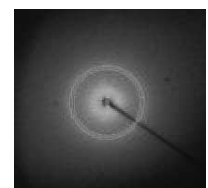

a.

b.

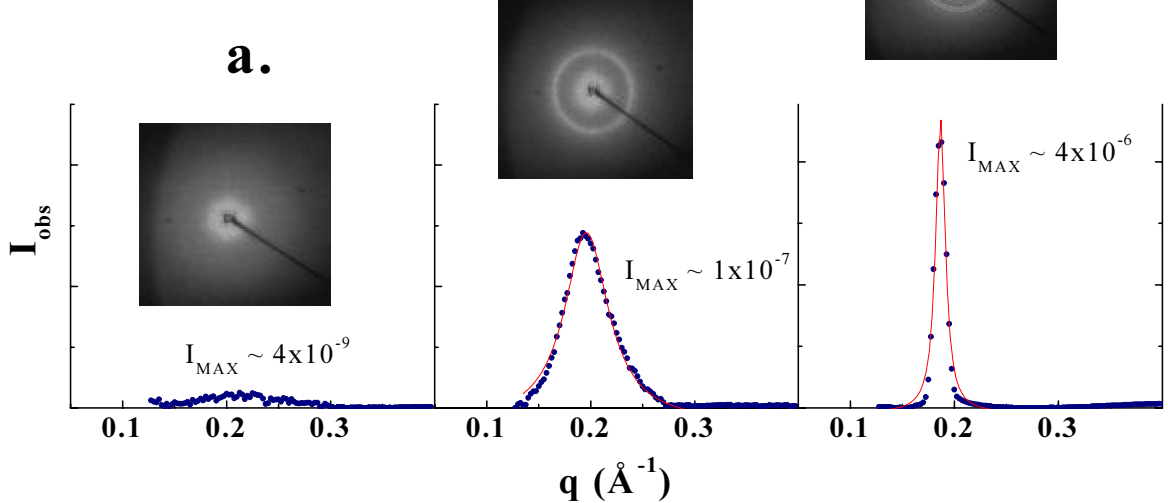

Figure 1. 2-D X-ray scattering spectra and 1-D intensity $I_{\text {obs }}(q)$ : experimental (ם), and Lorentzian function fitting (Eq. 1) (-) in the phases: (a) $I$; (b) $N^{*}$; (c) $A$. $I_{\max }$ corresponds to maximum intensity at peak position.

Figure 1 also presents the 1-D X-ray scattering related to the 2-D images. Note that the X-ray scattering peak intensity rises and the width becomes narrow as the positional order increases in the system.

\section{III.1.1 Distance between smectic planes and the mean molecular length}

Figure 2 (part I) presents the distance between the smectic $A$ planes $\left(d_{A}\right)$, and the experimental mean molecular length $(\bar{\ell})$ in the $N^{*}$ phase [14] as a function of the temperature ratio: $t=\left(T-T_{N^{*} A}\right) / T_{N^{*} A}[8]$.
Accordingly, $d_{A}$ is practically constant in the $A$ phase domain, whereas $\bar{\ell}$ decreases as temperature increases in $N^{*}$ phases. Table 1 shows the observed $d_{A}$ values in comparison with those calculated from the mean molecular structure $\left(l_{M}\right)$. Thus, one can note that the distance between the layers in the $A$ phase is circa $10 \%$ smaller than the corresponding mean molecular length calculated by assuming a total extended chain conformation. Moreover, $d_{A}$ decreases with $l_{M}$, as expected. 
(I)

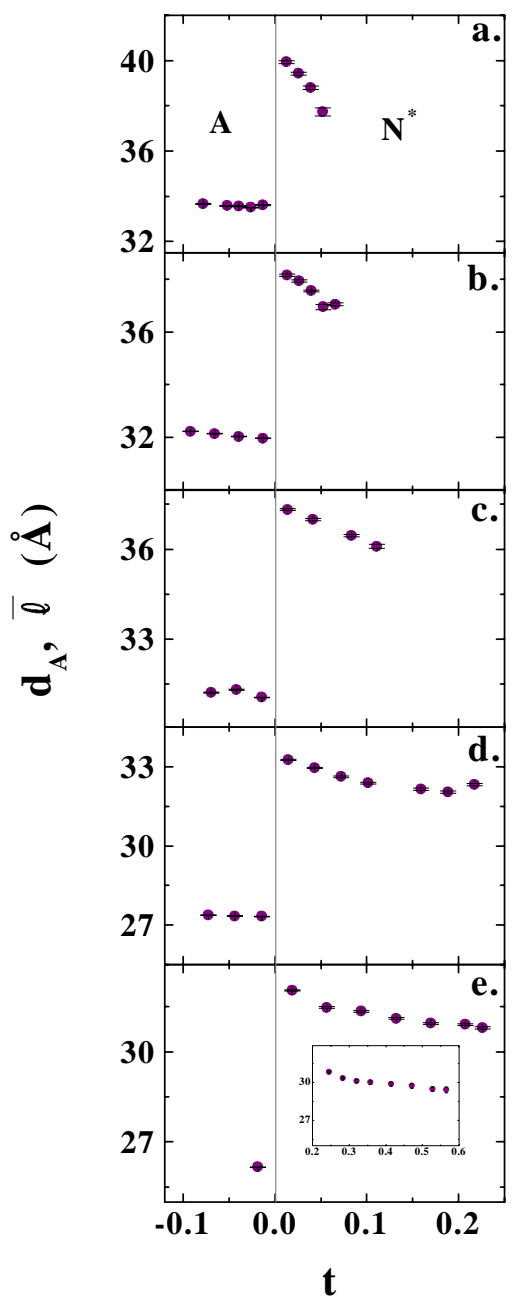

(II)

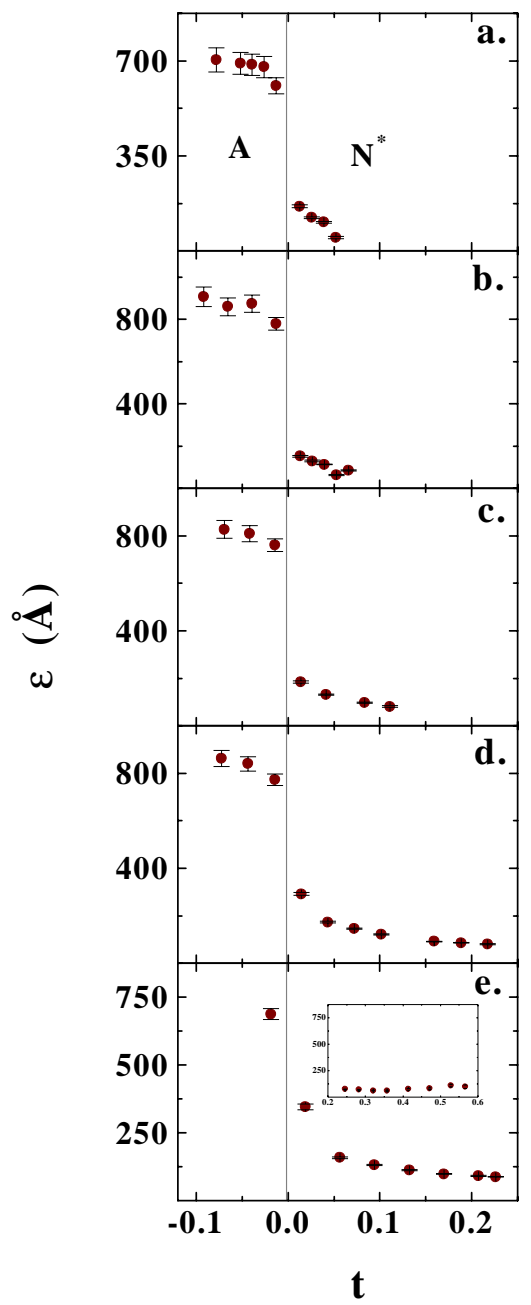

Figure 2. (I) distance $\left(d_{A}\right)$ between the smectic $A$ layers and mean molecular length $(\bar{\ell})$ in the $N^{*}$ phase; and (II) correlation length $(\varepsilon)$ (average values between cooling and heating cycles) as a function of the temperature ratio $t$ [8] for the following samples:(a) $C_{14}$; (b) $81.5 \mathrm{~mol} \%$ of $C_{14}$ in the $C_{9}-C_{14}$ mixture; (c) $63.5 \mathrm{~mol} \%$ of $C_{14}$ in the $C_{9}-C_{14}$ mixture; (d) $C_{9}$; (e) $63.1 \mathrm{~mol} \%$ of $C_{9}$ in the $C_{9}-C_{6}$ mixture. The inset in (e) shows $\bar{\ell}$ and $\varepsilon$ values for a greater interval of $t=\left(T-T_{A N^{*}}\right) / T_{A N^{*}}$ obtained for this sample within the $N^{*}$ phase.

On the other hand, the observed mean molecular length $\bar{\ell}$ in the $N^{*}$ phase near the $A-N^{*}$ phase transition $\left(\bar{\ell}_{A N^{*}}\right)$ is greater than $l_{M}$ (Table 1$)$. This behavior can be due to the fact that the expression to calculate $\bar{\ell}$, suggested by de Vries [14], is valid in $I$ and $N$ phases, when the molecules do not have a strong orientational order effect. Therefore, we believe that $\bar{\ell}_{I N^{*}}(\bar{\ell}$ in the $N^{*}$ phase near the $N^{*}-I$ phase transition) is, indeed, the mean molecular length, and $\bar{\ell}_{A N^{*}}$ has no meaning. Anyway, $d_{A}$ is always smaller than $\bar{\ell}_{I N^{*}}$ due to the contraction of the molecules as the temperature is reduced.

As previously mentioned, one can note from the Fig. 2 (part I) that the $A$ phase domain decreases and the $N^{*}$ phase increases as $l_{M}$ decreases.

\section{III.2 Correlation length}

Figure 2 (part II) shows the correlation length $(\varepsilon)$ in the $A$ and $N^{*}$ phases for all studied samples. As one can observe, $\varepsilon$ increases in $N^{*}$ phase as temperature decreases. This is due to an increase in the orientational order when the temperature moves away from the $I-N^{*}$ phase transition and approachs to the $N^{*}-A$ phase transition.

In contrast, the correlation length is practically constant in the $A$ phases with a small decrease in the $N^{*}-A$ phase transition vicinity, still in the $A$ phase domain. Regarding to $63.1 \mathrm{~mol} \% C_{9}$ in the $C_{9}-C_{6}$ binary mixture, just one temperature in the $A$ phase (Fig. 2.I-e) was experimentally obtained, because its domain is very narrow before the appearance of the crystal phase. Moreover, there is a clear discontinuity in the values of $\varepsilon$ along the $N^{*}-A$ phase transition (Fig. 2.II). However, the decrease in the mean molecular length is accompanied by a decrease in the corre- 
lation length discontinuity $(\Delta \varepsilon)$ at the $N^{*}-A$ phase transition. This because the correlation length in the $N^{*}$ phase near the $N^{*}-A$ phase transition is greater for samples with shorter mean molecular length bringing about a decrease in $\Delta \varepsilon$ in the referred phase transition.

\section{III.3 Viscosity}

The viscosity, $\eta$, results versus temperature ratio and shear rate, $\gamma$, near the $A-N^{*}$ phase transition, from the pure compounds $C_{14}$ and $C_{9}$ are shown in the figure 3. Note the presence of a peak [15, 16] at the $A-N^{*}$ phase transition for the $C_{14}$ pure compound (Fig. 3-a). Indeed viscosity data have provided a sensitive measurement for phase transitions[15] and, in this case, a clear distinction between the smectic $A$ and the cholesteric phases viscosity values. The magnitude of the peak as well as the viscosity values at $A$ phase decrease markedly with increasing shear rate. This might be correlated to the fact that it is easier to breakup the smectic structure as the shear rate is increased. Similar results for the same compound was obtained by Porter et al. [11].

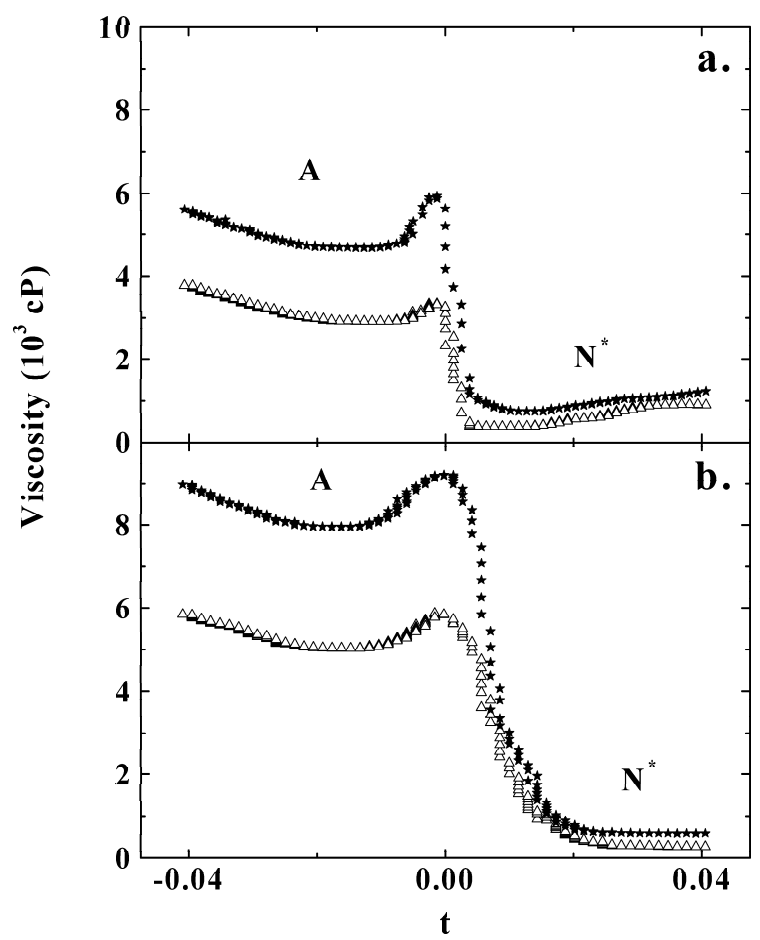

Figure 3. Viscosity vs temperature ratio $t=(T-$ $\left.T_{A N^{*}}\right) / T_{A N^{*}}$ at $A-N^{*}$ phase transition: (a) $C_{14}$ and (b) $C_{9}$. Shear rate $(\gamma): \star \rightarrow 1.5 \mathrm{~s}^{-1}$ and $\Delta \rightarrow 3.0 \mathrm{~s}^{-1}$.

The viscosity values increase at the $A-N^{*}$ phase transition for the $C_{9}$ while $r$ diminishes from 0.986 $\left(C_{14}\right)$ to $0.956\left(C_{9}\right)$ shown in figure 3 -b. On the other hand, the changes in the $\eta$ values are not sharp in $C_{9}$ as in $C_{14}$ compound from $N^{*}$ to $A$ phases $(\Delta t \approx 0.2$ for $C_{9}$ ). This $A-N^{*}$ phase transition has been considered as being weakly of first order by McMillan[1]. His X-ray experiments point up to the growth of smecticlike short-range order in the cholesteric phase near the $A-N^{*}$ phase transition. Our experimental results seem to be consistent with this fact. Such pretransition effect can be related to the increase in the viscosity values and reduced temperature interval observed in this phase transition.

Figure 4 shows the viscosity values at shear rate of $\gamma=0.4 s^{-1}$ for $63.1 \mathrm{~mol} \% C_{9}$ at $r=0.918$ in the $C_{9}-C_{6}$ system, where the existence of a possible second order $A-N^{*}$ phase transition was recently investigated[8]. Such low value of shear rate was used due to the strong increase in the viscosity values near the phase transition. Note that the viscosity for this mixture at $\gamma=0.4 s^{-1}$ is on the same order of magnitude observed for $C_{14}$ compound at $\gamma=3 \mathrm{~s}^{-1}$. Such increase in viscosity for the $C_{9}-C_{6}$ compound in the $N^{*}-A$ phase transition can be related to the small domain of the A phase in this mixture, as shown in the x-ray experiments, followed by a very near crystal phase. Moreover, the interval of temperature associated to the changes in the $\eta$ values from $N^{*}$ to $A$ is larger than for $C_{9}(\Delta t \approx 0.4$ for this mixture).

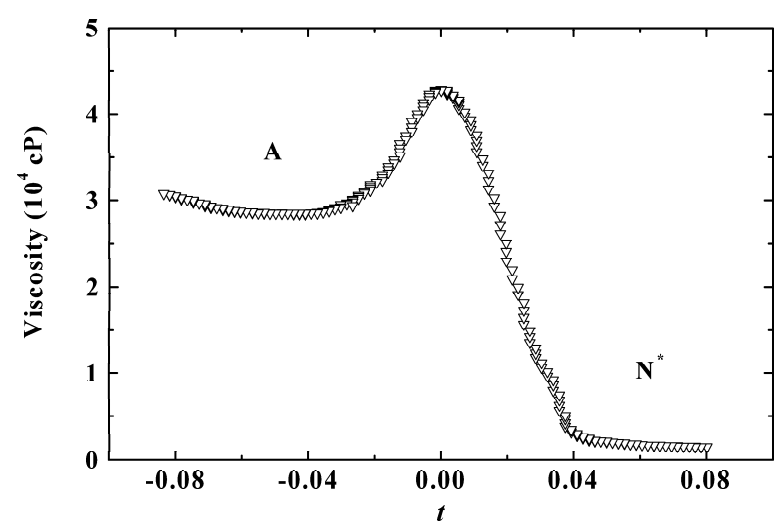

Figure 4. Viscosity vs temperature ratio $t=(T-$ $\left.T_{A N^{*}}\right) / T_{A N^{*}}$ at $A-N^{*}$ phase transition for the $63.1 \mathrm{~mol} \%$ of $C_{9}$ in the $C_{9}-C_{6}$ mixture $\left(\gamma=0.4 \mathrm{~s}^{-1}\right)$.

To sum up, the results obtained through X-ray and viscosity measurements used in this work, investigating the same liquid crystalline compounds of the homologous series of the cholesteryl $n$-alkanoates [8], present several interesting features in the nature of the $A-N^{*}$ phase transition, depending on the mean molecular length. As far as the X-ray scattering data are concerned, a decrease in $A$ phase domain and an expansion of the $N^{*}$ phase domain with the reduction of the $l_{M}$ were observed. This behavior is the same obtained theoretically by Lee et al. [3]. The distance between layers in the $A$ phase is compatible, although a little smaller, to the calculated $l_{M}$ and the experimental $\bar{\ell}_{I N *}$ mean molecular length. Moreover, $d_{A}$ decreases with $l_{M}$ as expected. The correlation length in the $N^{*}$ phase is 
greater for the samples with smaller mean molecular lengths. The viscosity values increases at the $A-N^{*}$ phase transition while $r$ diminishes. For $63.1 \mathrm{~mol} \%$ $C_{9}$ in the $C_{9}-C_{6}$ a big increase in the viscosity of the system was observed as $N^{*}-A$ phase transition is approached, where the existence of a possible second order phase transition was pointed out[8].

\section{Acknowledgments}

This work was supported by research grants from Pronex/MCT and FAPESP to R. Itri, Fundação Araucária and $\mathrm{CNPq}_{\text {to }} \mathrm{A}$. J. Palangana and M. Simões. E. L. Duarte and A. R. Sampaio had a master fellowship from FAPESP and CAPES, Brazil, respectively. Research fellowship to R. Itri from $\mathrm{CNPq}$ is also appreciated.

\section{References}

[1] W. L. McMillan, Phys. Rev. A, 6, 936 (1972); Phys. Rev. A, 4, 3, 1238 (1972).

[2] T.C. Lubensky, J. Phyique, 36, C1-151 (1975).

[3] F.T. Lee, H.T. Tan, Y.M. Shih, and C. W. Woo, Phys. Rev. Lett. 31, 1117 (1973).

[4] D. L. Johnson, C. Maze, Z. Oppenheim, and R. Reynolds, Phys. Rev. Lett. 34, 1143 (1975).
[5] J. Thoen, H. Marynissen, and W. Vandael, Phys. Rev. Lett. 52, 204 (1984).

[6] A.J. Palangana, S. Jayaraman, and T. Kroin, Mol. Cryst. Liq. Cryst., 131, 217 (1985).

[7] A.J. Palangana, L. R. Evangelista, M. Simões, and S. Jayaraman, Liq. Cryst., 15, 3, 377 (1993).

[8] E.L. Duarte, A.J. Palangana, R. Itri, A.R. Sampaio, and A.A. Barbosa, Eur. Phys. J. B 5, 835 (1998).

[9] J.H. Wendorff and F.P. Price, Mol. Cryst. Liq. Cryst. 22, 85 (1973).

[10] C. Tanford, J. Phys. Chem. 76, 3020 (1972); 78, 2469 (1974).

[11] K. Sakamoto, R.S. Porter, and J.F.Johnson, Mol. Cryst. Liq. Cryst. 8, 443 (1969).

[12] http://www.umh.ac.be/ poly/XRay/X-Ray.htm.

[13] D.E. Moncton and R. Pindak, Phys. Rev. Lett. 43, 10 (1979).

[14] A. de Vries, Mol. Cryst. Liq. Cryst. 10, 219 (1970); 11, 361 (1970); 20, 119 (1973).

[15] M. Kuzma, Y.W. Hui, and M.M. Labes, Mol. Cryst. Liq. Cryst. 172, 211 (1989).

[16] A.R. Sampaio, P.R.G. Fernandes, M. Simoes, and A.J. Palangana, Mol. Cryst. Liq. Cryst. 359, 269 (2001).

[17] D. Demus, H.G. Hahn, and F. Kuschel, Mol. Cryst. Liq. Cryst. 44, 61 (1978) 